\title{
Research and Development of the SC230 Superconducting Cyclotron for Proton Therapy
}

\author{
O. V. Karamyshev ${ }^{a}$ *, K. S. Bunyatov ${ }^{a}$, A. L. Gibinsky ${ }^{a}$, S. V. Gurskiy ${ }^{a}$, G. A. Karamysheva ${ }^{a}$, \\ I. D. Lyapin ${ }^{a}$, V. A. Malinin ${ }^{a}$, D. V. Popov ${ }^{a}$, G. D. Shirkov ${ }^{a}$, and S. G. Shirkov ${ }^{a}$ \\ ${ }^{a}$ Joint Institute for Nuclear Research, Dubna, 141980 Russia \\ *e-mail: olegka@jinr.ru
}

Received September 4, 2020; revised September 17, 2020; accepted September 17, 2020

\begin{abstract}
This paper presents a conceptual design of the compact SC230 superconducting cyclotron that accelerates a beam of protons to $230 \mathrm{MeV}$ and is intended for proton therapy and biomedical research. SC230 is an isochronous four-sector cyclotron based on a shell-type magnet with a magnetic field in the center of 1.7 T. Superconducting coils will be enclosed in a cryostat; all other parts of the cyclotron are warm. Acceleration is carried out at the fourth harmonic mode of the accelerating high-frequency (HF) system consisting of four resonators located in the cyclotron valleys. The accelerator employs an internal Penning-type hot cathode source. Extraction is carried out using an electrostatic deflector located in the gap between the sectors and two passive magnetic channels.
\end{abstract}

DOI: $10.1134 / \mathrm{S} 1547477121010088$

\section{INTRODUCTION}

Using beams of protons and nuclei of light elements in radiation therapy for cancer, for a number of reasons, is more preferable than irradiation with gamma quanta and beams of accelerated electrons. This is mainly due to the more favorable distribution of the absorbed dose in the depth of penetration of charged particles into the irradiated region. This method is especially important for pediatric oncology, as well as in cases where the tumor is located near critical organs or has a complex shape. The use of proton and hadron beams is recognized all over the world as an effective way to treat oncology.

At the Joint Institute for Nuclear Research (JINR), the first sessions of clinical application of proton beams generated by Phasotron were started in 1967 $[1,2]$. The Phasotron beam was used to implement the technique of three-dimensional conformal proton radiation therapy; it was the first one in Russia. From 2000 to 2018, about 1300 patients with various neoplasms underwent proton radiation therapy.

JINR, having many years of experience in creating accelerators and physics facilities for fundamental and applied research, develops medical accelerator equipment in cooperation with IBA, the global leader in the field of creating devices for proton therapy. JINR specialists carried out calculations and modernization of the serial proton cyclotron C235. The first copy of C235-V3 was assembled, adjusted, and launched at JINR in 2012 [3]. Currently, C235-V3 is part of the Federal High-Tech Center for Medical Radiology of
FMBA of Russia in Dimitrovgrad. JINR and IBA have jointly developed the project of the world's first superconducting carbon cyclotron C400 [4], which is currently being built in Caen, France.

At present, the Dzhelepov Laboratory of Nuclear Problems (DLNP) is developing its own project of an isochronous cyclotron intended for treating cancer patients. Proton therapy requires the acceleration of the proton beam to energies of $60-230 \mathrm{MeV}$. There are various ways to achieve such proton accelerations. Today, medical centers employ three types of accelerators: cyclotrons, synchrocyclotrons, and synchrotrons. Linear accelerators for proton therapy are also being developed. Each of these types of accelerators has its own advantages and disadvantages.

Thus, the advantages of cyclotrons include its high reliability and simplicity of the accelerator, which employs a constant magnetic field, an accelerating system with a constant frequency, and a high beam current; cyclotrons allow for accurate and fast modulation of the beam intensity. A disadvantage is the extraction of a single fixed energy; therefore, to obtain lower energies, one has to use an energy selection system: a degrader that deteriorates beam parameters. Synchrotrons have a high beam quality, but their beam current is orders of magnitude lower. The need to use a booster results in relatively large sizes and costs of synchrotrons. Synchrocyclotrons have the same disadvantages as cyclotrons, and the pulsed nature of their acceleration results in low average beam intensities. However, superconducting synchrocyclotrons 
Table 1. Comparison of significant parameters of cyclotrons that lead in the proton therapy market and the SC230 project

\begin{tabular}{|c|c|c|c|}
\hline Parameter & Varian Proscan & IBAC235 & SC230 \\
\hline Width, m & 3.1 & 4.3 & 3.5 \\
\hline$B_{0}, \mathrm{~T}$ & 2.4 & 1.7 & 1.7 \\
\hline Coil & NbTi & Copper & NbTi or HTSC? \\
\hline Weight, $\mathrm{t}$ & 90 & 200 & 94 \\
\hline $\mathrm{HF}$ generator frequency, $\mathrm{MHz}$ & 72.8 & 106 & 106 \\
\hline HF system power, $\mathrm{kW}$ & 115 & 60 & 55 \\
\hline Number of resonators & 4 & 2 & 4 \\
\hline Acceleration harmonic mode & 2 & 4 & 4 \\
\hline
\end{tabular}

allow the use of high magnetic fields, providing ultracompact solutions, which led to a growing demand for such devices in the proton therapy market.

An isochronous cyclotron accelerates a quasi-continuous beam; therefore it is the most promising accelerator for the application of a new method of radiation therapy, FLASH. FLASH-RT consists of delivering the required radiation dose at ultrahigh dose rates. In contrast to the radiation therapy carried out with conventional dose rates $(1-7 \mathrm{~Gy} / \mathrm{s})$, FLASH irradiation is carried out at dose rates over $40 \mathrm{~Gy} / \mathrm{s}$ in less than $0.5 \mathrm{~s}$. Healthy tissue resists FLASH radiation better, while tumors have the same levels of sensitivity as in conventional treatment. Health specialists are highly interested in this method, since it not only reduces the effect on healthy tissues, but also reduces the number of treatment procedures from 10-30 in the case of conventional treatment to $1-3$. However, before proton FLASH therapy can be fully implemented in practice (preclinical studies are currently underway), especially for dose delivery through pinpoint scanning, which is important for ensuring high-precision dose contours, several engineering problems need to be solved. In particular, an accelerator is needed that provides an average beam current amounting to, according to various estimates, $0.5-2 \mu \mathrm{A}$ for the entire range of energies used in treatment $[5,6]$.

Currently developed projects of cyclotrons for proton therapy are aimed at making the cyclotron as compact as possible, reducing its size and weight by increasing the magnetic induction. The newest superconducting cyclotrons being created for proton therapy, such as SC200 (the SC200 project was developed at JINR jointly with ASIPP, Hefei, China) [7], PronovaK230 (United States) [8], and Sumitomo $230 \mathrm{MeV}$ [9], have similar parameters that determine the structure of the cyclotron. These projects are four-sector isochronous cyclotrons based on a shell-type magnet with a magnetic field in the center of $\sim 3 \mathrm{~T}$. None of these cyclotrons have been put into operation yet, mainly due to the multiple engineering difficulties arising from the high magnetic field of the accelerator. When the magnetic field reaches $3 \mathrm{~T}$, cyclotrons become more difficult to set up and require much more precision in manufacturing, which increases the costs of the project; that is, they lose some of the advantages of this type of accelerators, while in terms of compactness they are still inferior to synchrocyclotrons.

In the field of proton therapy, the two most successful operating accelerators are the Varian Proscan superconducting cyclotron [10] and the C235 warm cyclotron (IBA, Belgium) [11]. Varian and C235 were designed in the 1990s; advances in simulation, superconducting technology, and HF generators are now making it possible to create significantly cheaper, more reliable, and compact devices. Both of these cyclotrons have lower magnetic fields in the center than modern designs, 2.4 and $1.7 \mathrm{~T}$, respectively.

Therefore, when working on our project, we decided to choose a low magnetic field of $1.5-1.7 \mathrm{~T}$ in the center. The corresponding frequency of the HF system for these values of the magnetic-field induction is $91-106 \mathrm{MHz}$ when operating at the 4th harmonic mode (see Table 1).

Calculations have shown that a twofold decrease in the magnetic induction of the field in comparison with SC200 and other modern projects will lead to a twofold increase only in the diameter of the pole, while the thickness of the cyclotron yoke, provided that the magnetic induction in the yoke is preserved (that is, provided the same magnitude of the scattered field around the accelerator), almost will not change.

Since the cyclotron will have a relatively small magnetic field, both superconducting and resistive coils can be used. Both of the solutions have their pros and cons and differ somewhat in parameters and design features. Possible versions of the concept of a cyclotron with a low mean field were presented at accelerator conferences [12, 13], published [14], and discussed and recommended for production at an expert meeting in Hefei. In this paper, we present a version of a compact isochronous cyclotron with a SC230 superconducting coil and a field level of $1.7 \mathrm{~T}$ whose overall dimensions and weight are comparable to the Varian Proscan cyclotron. 
Table 2. Parameters of the SC230 cyclotron

\begin{tabular}{l|c}
\hline \multicolumn{1}{c|}{ Magnet type } & SC coil, warm yoke \\
\hline Final energy, MeV & 230 \\
Average magnetic field (center/terminal), T & $1.7 / 2.15$ \\
Dimensions (height $\times$ width), $\mathrm{m}$ & $1.7 \times 3.5$ \\
Weight of magnet, $\mathrm{t}$ & 94 \\
Hill/valley gap, mm & $50 / 700$ \\
Ampere-turns & 310000 \\
SC coil weight, $\mathrm{kg}$ & 170 \\
Current density in the coil, A/cm ${ }^{2}$ & 90 \\
HF system frequency, MHz & 106 \\
Acceleration harmonic mode & 4 \\
Number of accelerating resonators & 4 \\
Voltage, center/extraction zone, $\mathrm{kV}$ & $30 / 120$ \\
HF system power, $\mathrm{kW}$ & 55 \\
Extraction radius, mm & 1070 \\
Number of revolutions & 500 \\
Beam intensity, $\mu \mathrm{A}$ & 1.0 \\
Extraction system type & ESD $+2 \mathrm{MC}$ \\
\hline
\end{tabular}

In our project, we have combined advantages of both the cyclotrons-leaders in the proton therapy market. From the $\mathrm{C} 235$ cyclotron, we took a low magnetic field and the fourth harmonic mode; similar to the Varian cyclotron, we used a superconducting coil and a constant gap between the sectors along the radius that is sufficient to house an electrostatic deflector and, as a result, valleys that are free to accommodate four accelerating resonators.

We plan to use a standard, widely used technology based on NbTi for the production of superconducting coils; however, the cyclotron project aimed at minimizing the energy consumption makes the use of hightemperature superconductors (HTSCs) promising due to the relatively small amount of the material required to provide a low coil current. The use of HTSCs is promising not only because of the potential use of cheap nitrogen for cooling, but also due to the use of helium as a coolant, since the wide temperature range up to the quench temperature will significantly simplify the cryogenic system and reduce operating costs.

The development of technologies in the field of superconductivity and the demand for a high beam intensity for FLASH-RT makes it interesting to develop a cyclotron that accelerates $\mathrm{H}^{2+}$ and carbon ions, as was suggested by L. Calabretta [15], but such an accelerator will have significantly larger dimensions, will require higher vacuum, and will result in multiple engineering difficulties due to the inevitable increase in the average field of the magnetic system. The relevance of such a project will be determined by the still unformed requirements for the beam parameters in FLASH-RT.

\section{SC230 CYCLOTRON}

The SC230 cyclotron can produce a $230-\mathrm{MeV}$ proton beam for therapy and biomedical research. Table 2 summarizes the main parameters of the SC230 cyclotron and Fig. 1 shows its computer model.

Computer simulation of a magnet. Calculations of the cyclotron magnet were carried out in CST Studio using a parameterized model (see Fig. 1) created in Autodesk Fusion 360.

Figure 1 shows the interior of the magnetic and accelerating systems of the cyclotron. The shape of the coil, cryostat, pole, and yoke follows the shape of the particle trajectory. The center of the cyclotron contains an internal Penning-type proton source. A low magnetic field in the center of $\sim 1.7 \mathrm{~T}$ will allow using a hot cathode source and provide a spacious central area sufficient to accommodate the necessary elements, such as orbit correction coils and phase-selection diaphragms. It is possible to place two sources of protons or use the axial injection of a beam generated by an intense external source when it is necessary to increase the intensity of the extracted beam, for example, for FLASH-RT.

The dimensions of the yoke (Figs. 1, 2) were chosen in such a way as to limit the scattered magnetic field in the range of $500 \mathrm{G}$ near the accelerator while avoiding the presence of regions of unsaturated iron (see Fig. 3). 


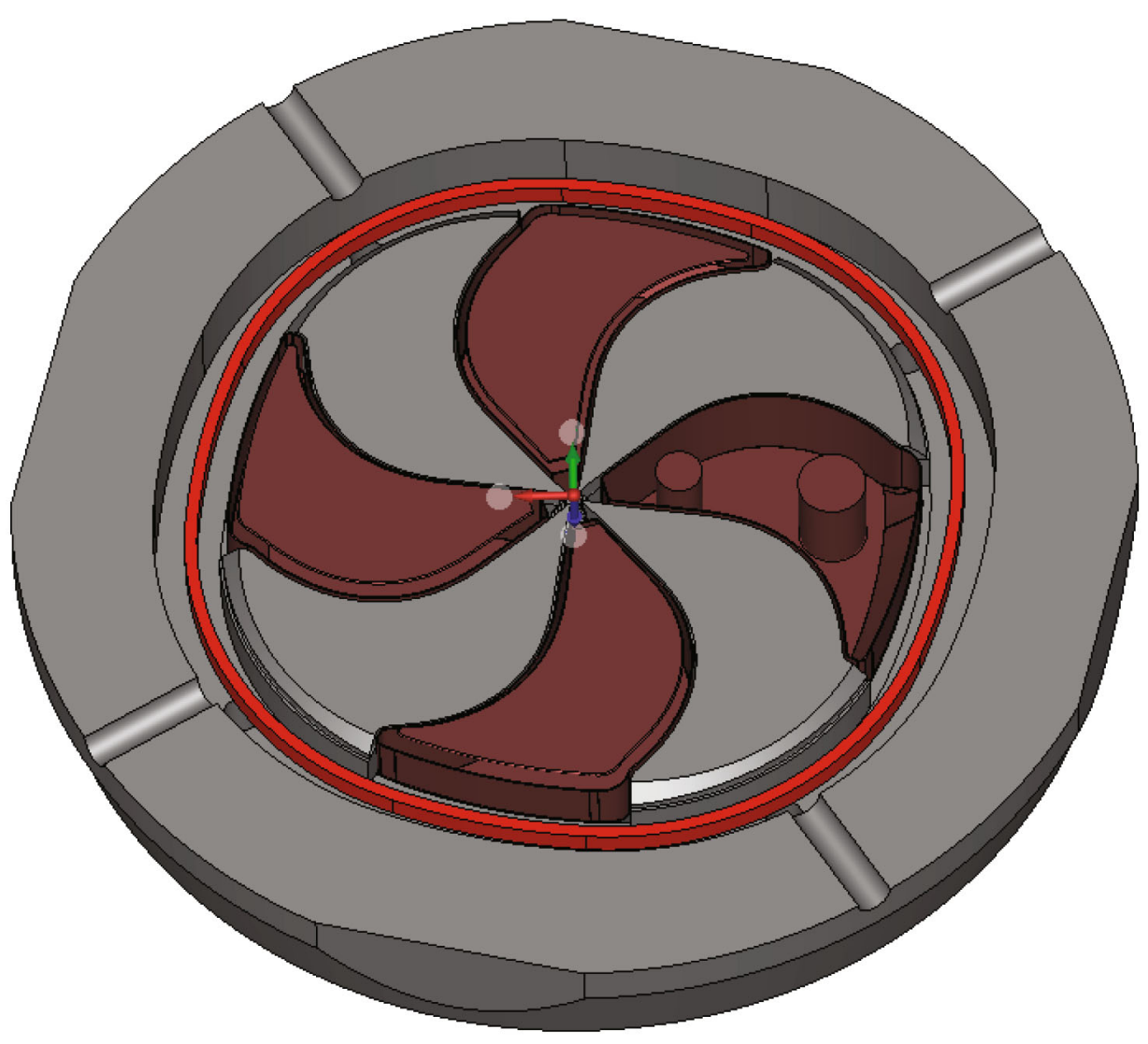

Fig. 1. Three-dimensional computer model of the cyclotron (the magnet and accelerating system).

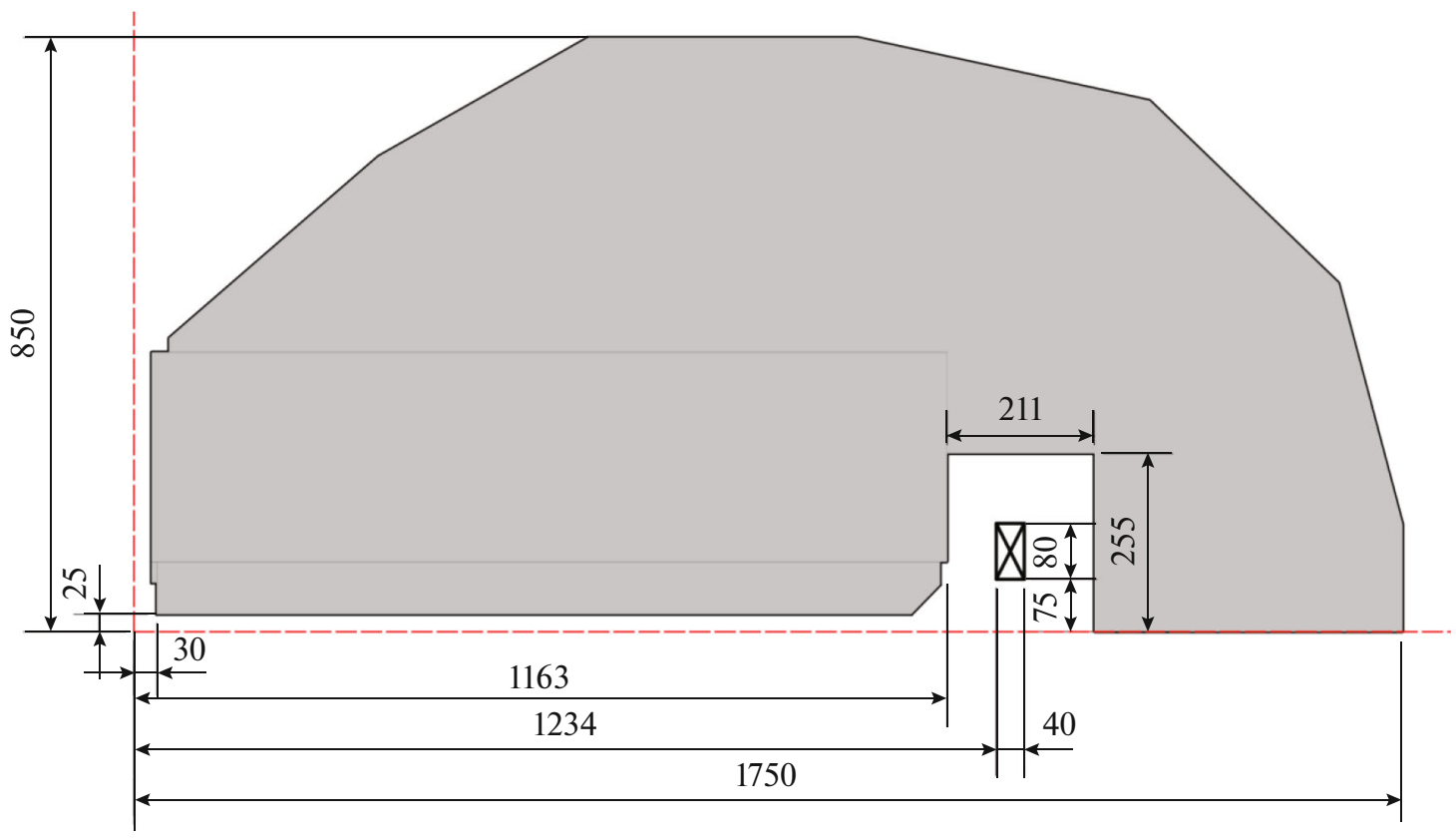

Fig. 2. Overall dimensions of the magnetic yoke and coil of SC230 (a quarter of the accelerator is shown). 


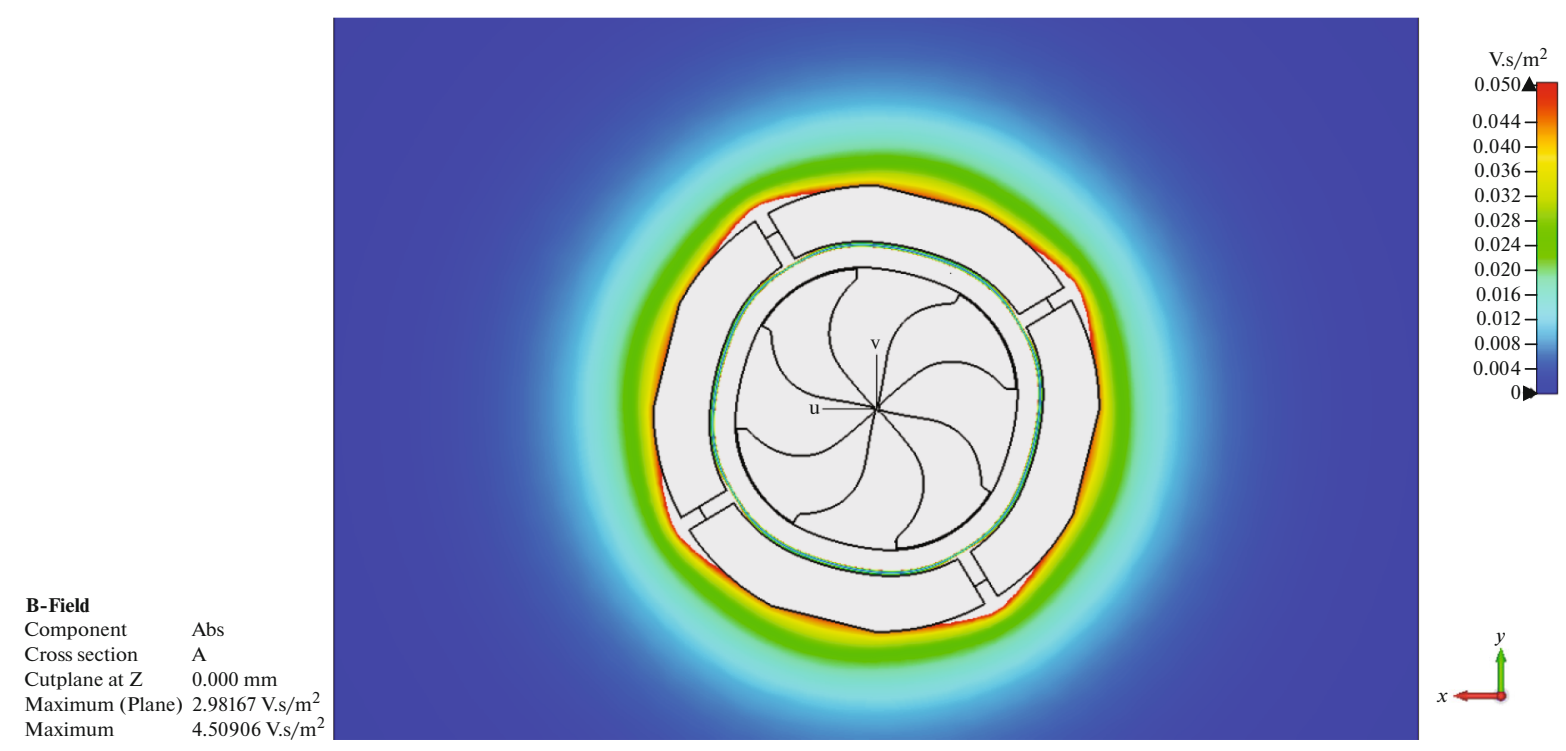

Fig. 3. Scattered magnetic field of the cyclotron in the median plane (red is $500 \mathrm{G}$, blue is $0 \mathrm{G}$, and white corresponds to the region in which the magnetic field is above $500 \mathrm{G}$ ).

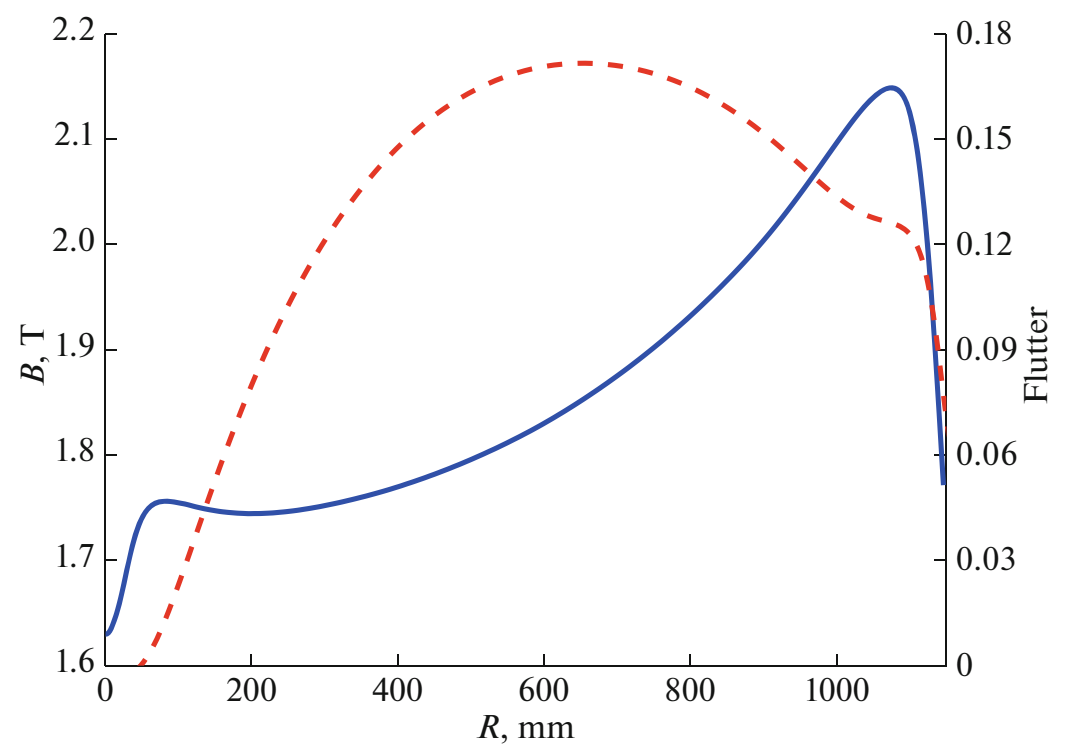

Fig. 4. Average magnetic field (blue solid line) and flutter (red dashed line), depending on the radius.

The average magnetic field and flutter of the developed model (a flutter characterizes the depth of azimuthal variation of the magnetic field) are shown in Fig. 4. A high flutter minimizes the helicity angle of the sector shims (see Fig. 5). The flutter and angular helicity of the sectors provide the vertical focusing necessary for beam acceleration (see the vertical frequency of betatron oscillations in Fig. 7).

As a rule, the most difficult task in the formation of the field of an isochronous cyclotron is to provide the growth of the mean field as far as possible along the pole radius in order to reduce the radial distance traveled by the beam when extracted from the high magnetic field, which facilitates the beam extraction and reduces the size and weight of the accelerator. This task is accomplished in various ways. For example, in the SC200 and C235 (IBA) cyclotrons, along with a higher azimuthal angle of the sectors, a profiled vertical gap between the sectors (elliptical shape) is used. In our case, the value of the vertical gap between the sectors is constant along the radius, $50 \mathrm{~mm}$, which is sufficient to accommodate an electrostatic deflector. To ensure the growth of the mean field in the 


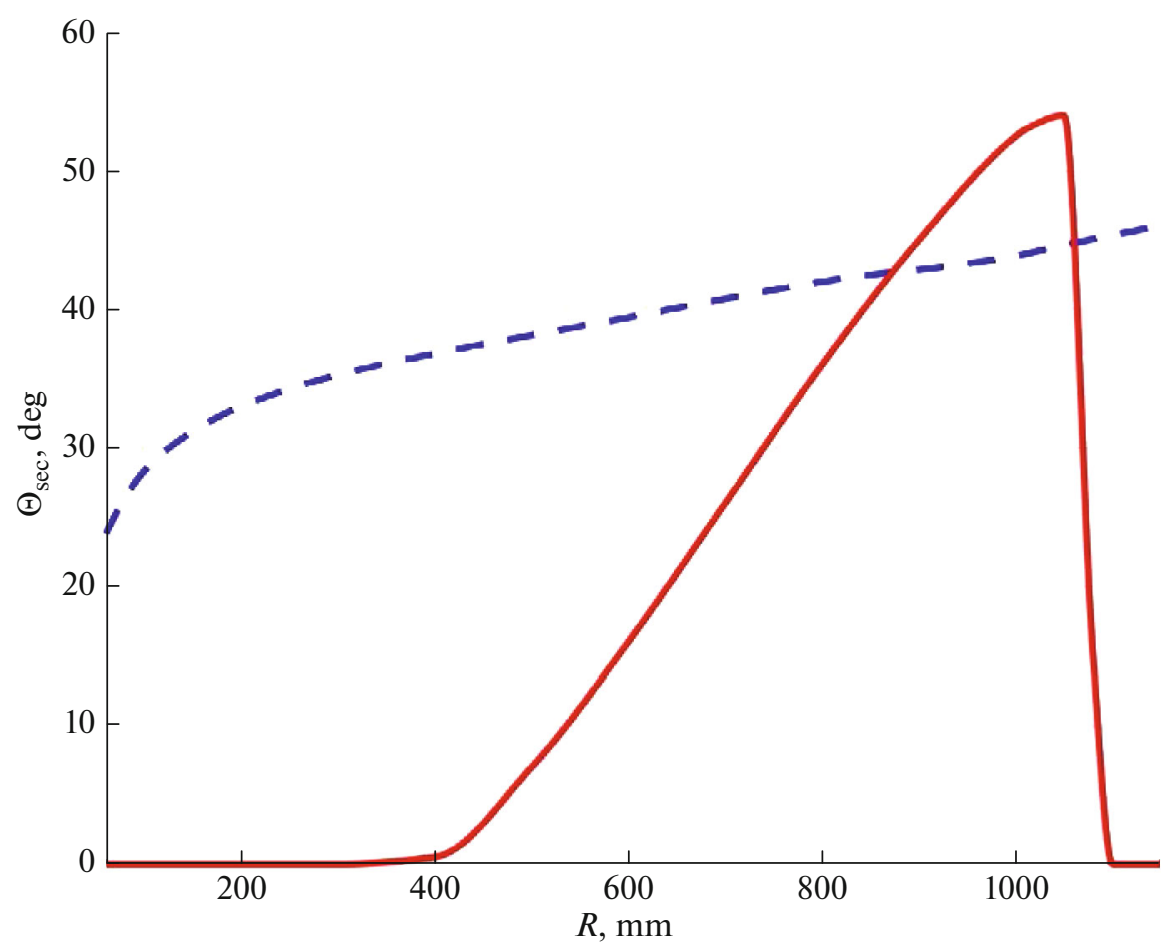

Fig. 5. Helicity angle of the sectors (red solid line) and the azimuthal width of the sector (blue dashed line), depending on the radius.

extraction zone, we set the shape of the pole not along a circle centered at the geometric center of the cyclotron but according to the shape of the trajectory of the accelerated particles, which differs from the circle more and more the higher the flutter of the magnetic field is. The shape of the superconducting coils is also replaced with a shape consisting of a sequence of cir-

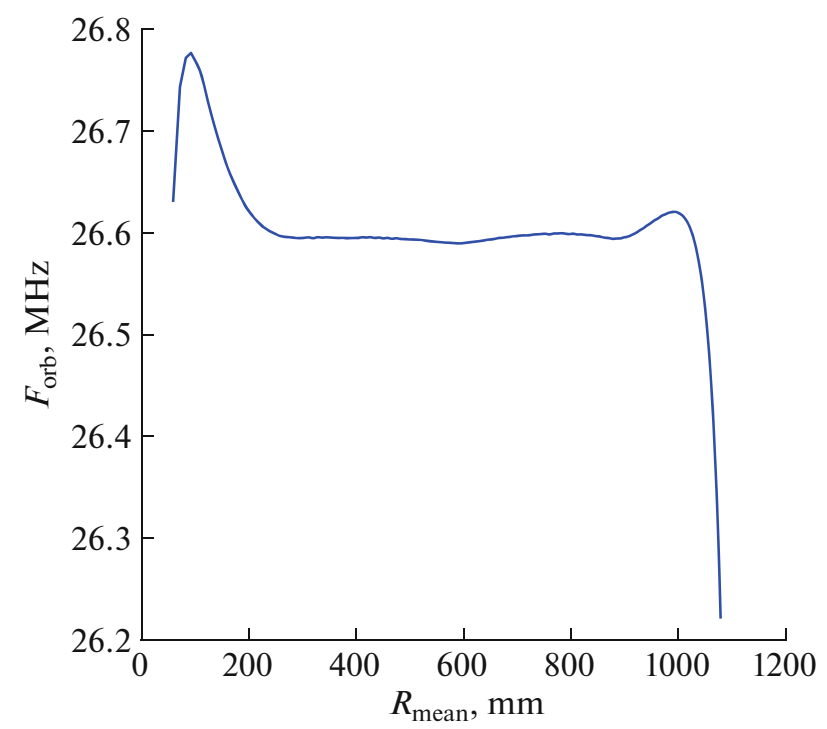

Fig. 6. Frequency of revolution of protons, depending on the radius. cular arcs approaching the trajectory of a particle. This shape of the coil allows for the most efficient use of the entire generated flux of magnetic induction, passing it exclusively through the beam-acceleration region, which saves the required number of ampere turns.

For the correct formation of the magnetic field in an isochronous cyclotron, it is necessary to provide the same revolution frequency of particles in closed equilibrium orbits for the entire range of radii and the travel of the operating point on the frequency diagram without crossing dangerous resonances. For this purpose, the magnetic-field map is analyzed using the CYCLOPS algorithm [16], one of the most reliable tools for analyzing particle dynamics in cyclotrons. There are multiple CYCLOPS code modifications intended for various specific applications (see $[17,18])$. We have developed our own versions of the implementation of algorithms based on CYCLOPS using the Matlab package. We started with the implementation of the classical CYCLOPS method with energy steps, the algorithm of which was implemented in Simulink, where convenient and visual debugging is possible. This version is used for various computational magnetic field maps. In addition, an algorithm from [18] was implemented, designed to search for equilibrium orbits for a given radius in the cyclotron. A program similar to the CYCLOPS modification proposed in [18] was also created; however, due to the choice of a slower method of convergence of the iterative procedure, it was possible to achieve a more stable 


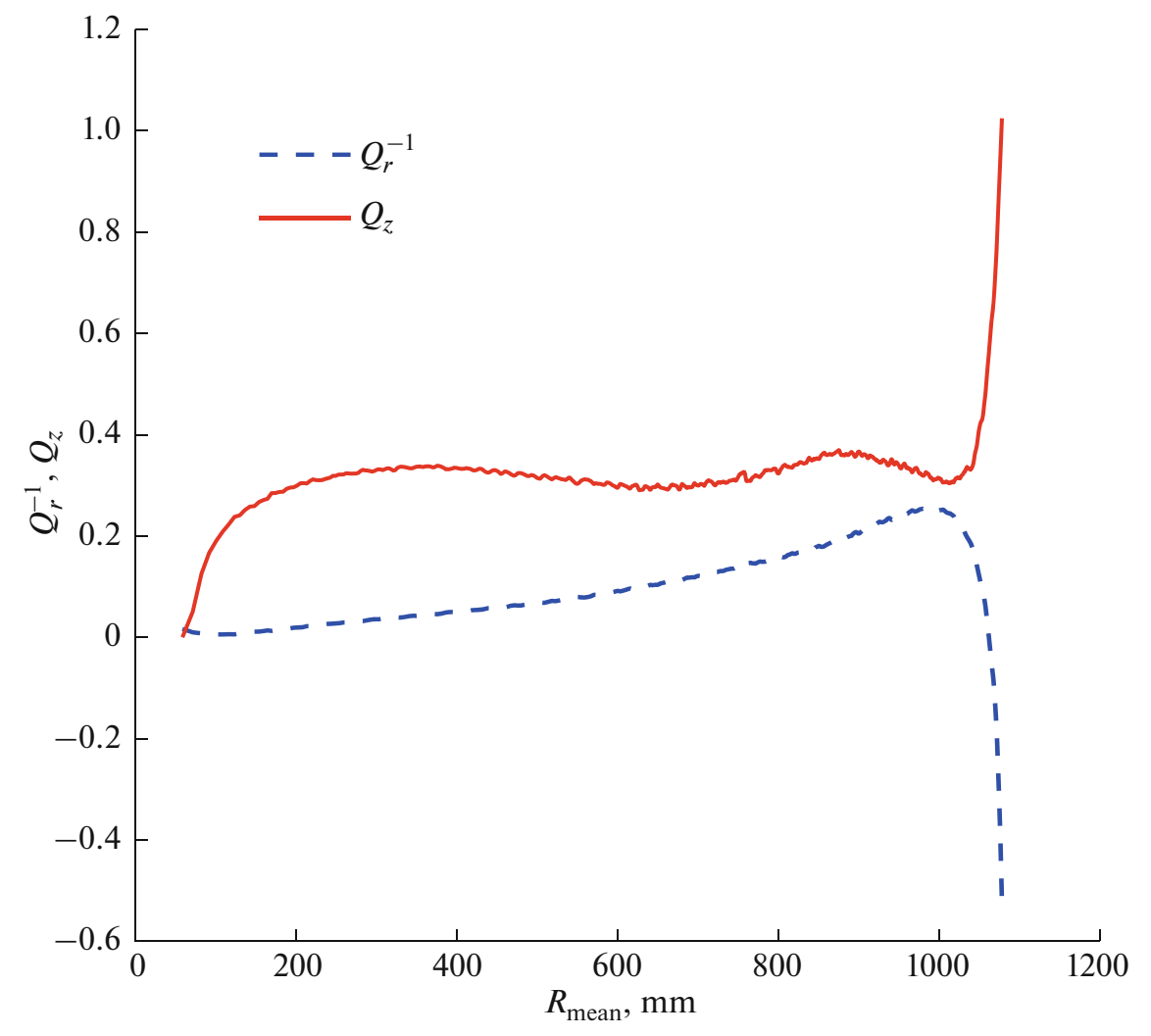

Fig. 7. Vertical and radial frequencies of betatron oscillations, depending on the radius.

operation of the code on maps with a lot of noise. This program contains an algorithm for finding equilibrium orbits and for calculating betatron frequencies. The calculation results can be used not only for the isochronization of the magnetic-field map, but also as input parameters for $3 \mathrm{D}$ modeling of the beam dynamics.

The algorithm for finding equilibrium orbits takes, as an input, a two-dimensional map of the magnetic field in the median plane. The magnetic-field map is preprocessed and smoothed; then, to increase the speed of work, it is accessed using the griddedInterpolant class in Matlab. The equations of motion are integrated using the 4th order Runge-Kutta method. This implementation makes it possible to simultaneously work with a large number of particles, which significantly increases performance. The frequency of revolution of particles and betatron frequencies calculated in the program developed using the CYCLOPS algorithm are shown in Figs. 6 and 7.

Project of the accelerating system. The accelerating high-frequency system is designed to transfer the energy of the accelerating electromagnetic field to the accelerated protons. The accelerating system of the SC230 cyclotron consists of four resonators and HF power supply, stabilization, and control systems. The resonators of the HF system are located in the valleys of the electromagnet poles; the geometry of the
HF system resonators is limited by the size of the spiral sectors.

To accurately calculate the parameters of the resonators of the accelerating system of the cyclotron, the CST MICROWAVE STUDIO (CST MWS) software was used, which is intended for the numerical simulation of high-frequency devices (antennas, filters, and resonators). It is particularly important for our calculations that CST allows using Perfect Boundary Approximation (PBA). A model of an accelerating system consisting of four half-wave resonators with two supports providing the required rigidity of the structure, which are connected in the center, is shown in Fig. 1. In one of the resonators, for better visualization, only the resonator and two supports are shown; the accelerating dee electrode is not shown.

When developing a model of the HF system, we determined the geometric dimensions of the resonator and the position and dimensions of the supports so as to provide the required frequency of $106 \mathrm{MHz}$ and the distribution of the accelerating field, which is used in the form of a field map in calculating the dynamics of particles.

The computational map of the accelerating field made it possible to significantly expand the information content of the analysis of the motion of an equilibrium particle in closed orbits, supplementing the 


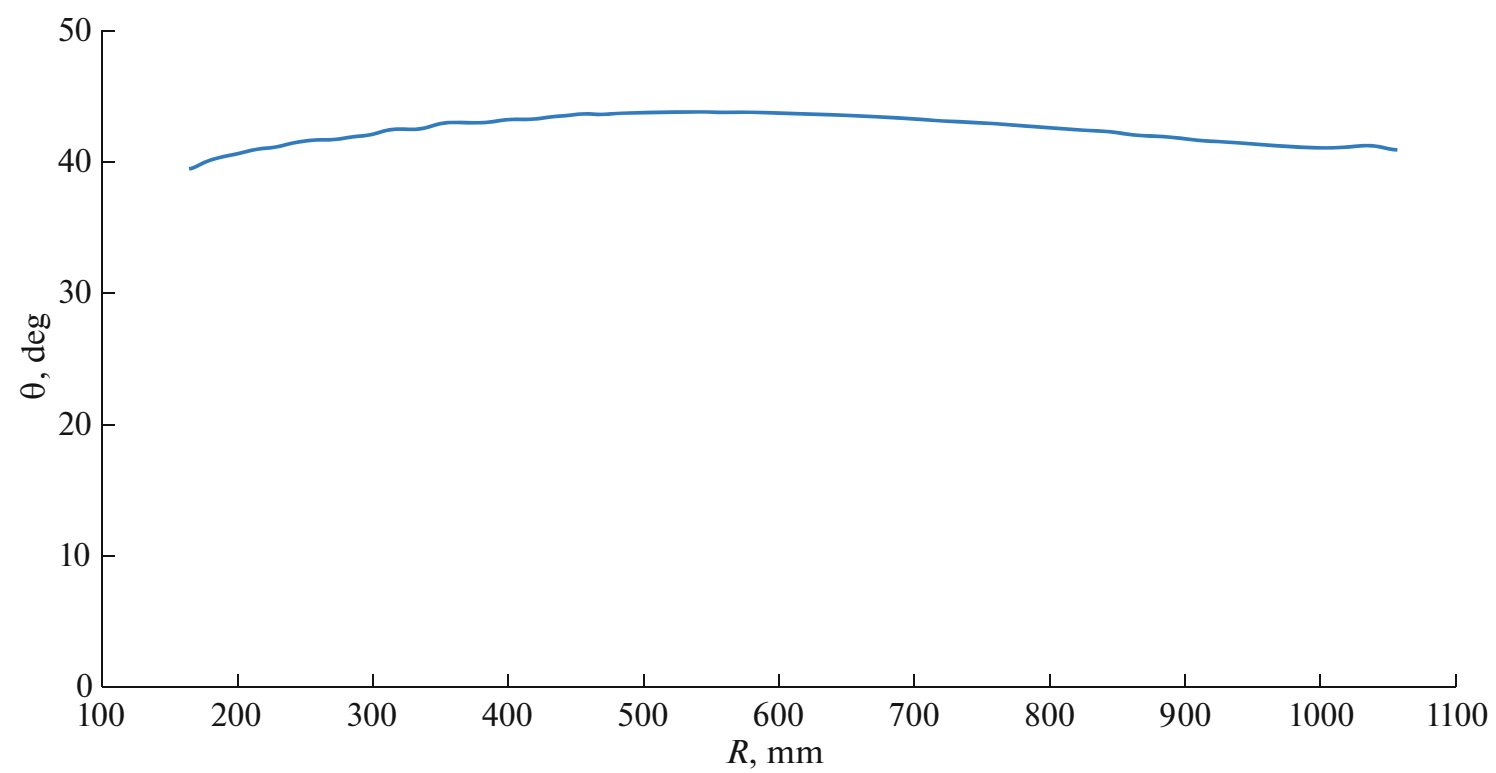

Fig. 8. Azimuthal of the resonator.

Matlab CYCLOPS+ code with the calculation of several useful characteristics.

The code calculates the effective azimuthal angle of the resonators, which is determined as a distance along the azimuthal angle between the maxima of the electric-field distribution in the median plane for the entire range of radii. As is seen from Fig. 8, the azimuthal angle of the resonator is about $40-44^{\circ}$.

Since the azimuthal angle of the resonator is close to optimal $\left(45^{\circ}\right.$ for acceleration at the 4 th harmonic mode), we assumed that the magnetic field generated by the HF system would not have a noticeable effect on the beam, so only the electric-field map was used to analyze the particle dynamics.

Let us define the voltage on the accelerating gaps as an integral of the electric-field strength along the arc of the circle passing through the gap. The voltage in the first and second gaps of the accelerating resonator along the course of the beam (the planned direction of beam acceleration is the direction of helicity of the sector shims), as well as the voltage averaged over two gaps on the resonator, depending on the radius, are shown in Fig. 9.

Thus, the developed model of the HF system has an accelerating voltage growing towards the extraction zone, which is preferable for increasing the step of the orbits and creating the effect of phase compression at finite radii.

Let us determine the maximum gain of energy per revolution depending on the radius of the accelerator:

$$
\Delta E_{\text {turn }}(R)=N_{\text {cav }}\left(V_{1}(R)+V_{2}(R)\right) \sin \left(h \frac{\theta_{\text {cav }}(R)}{2}\right)
$$

where $N_{\text {cav }}=4$ is the number of accelerating resonators; $V_{1}(R), V_{2}(R)$ is the voltage variation along the radius in the first and second accelerating gaps (Fig. 9); $h=4$ is the acceleration harmonic mode; and $\theta_{\text {cav }}$ is the azimuthal angle of the resonator (Fig. 8). The calculation result is shown in Fig. 10.

The results of the magnetic-field analysis provide information about the phase of the equilibrium particle in each orbit. Traditionally, the phase motion is estimated on the basis of the assumed energy gain per revolution, the same for each revolution, which is permissible if the accelerating voltage changes only slightly along the radius and the width of the resonator throughout the entire acceleration cycle is optimal. In real cyclotrons, both conditions are rarely fulfilled; therefore, an accurate calculation of the phase motion can be obtained in the process of beam tracing in computational maps. The Matlab CYCLOPS+ code was supplemented with the calculation of phase motion when using the accelerating field map. The integral phase shift is considered in the form of an iterative procedure that sequentially sums up the increment in the phase shift and the energy gain for each accelerating slit on the basis of the calculated dependences of the azimuthal angle and resonator voltages on energy.

The phase motion of an equilibrium particle corresponds to the number of revolutions in the accelerator performed by the particle. In the given magnetic and electric fields, an equilibrium particle reaches a final energy of $230 \mathrm{MeV}$ in less than 500 revolutions. Figure 12 shows the step of the orbits and the number of revolutions depending on the radius; the result of calculating the phase motion by the analysis of equilibrium orbits is shown in Fig. 11 as a black bold line. Thus, the Matlab CYCLOPS + code gives complete 


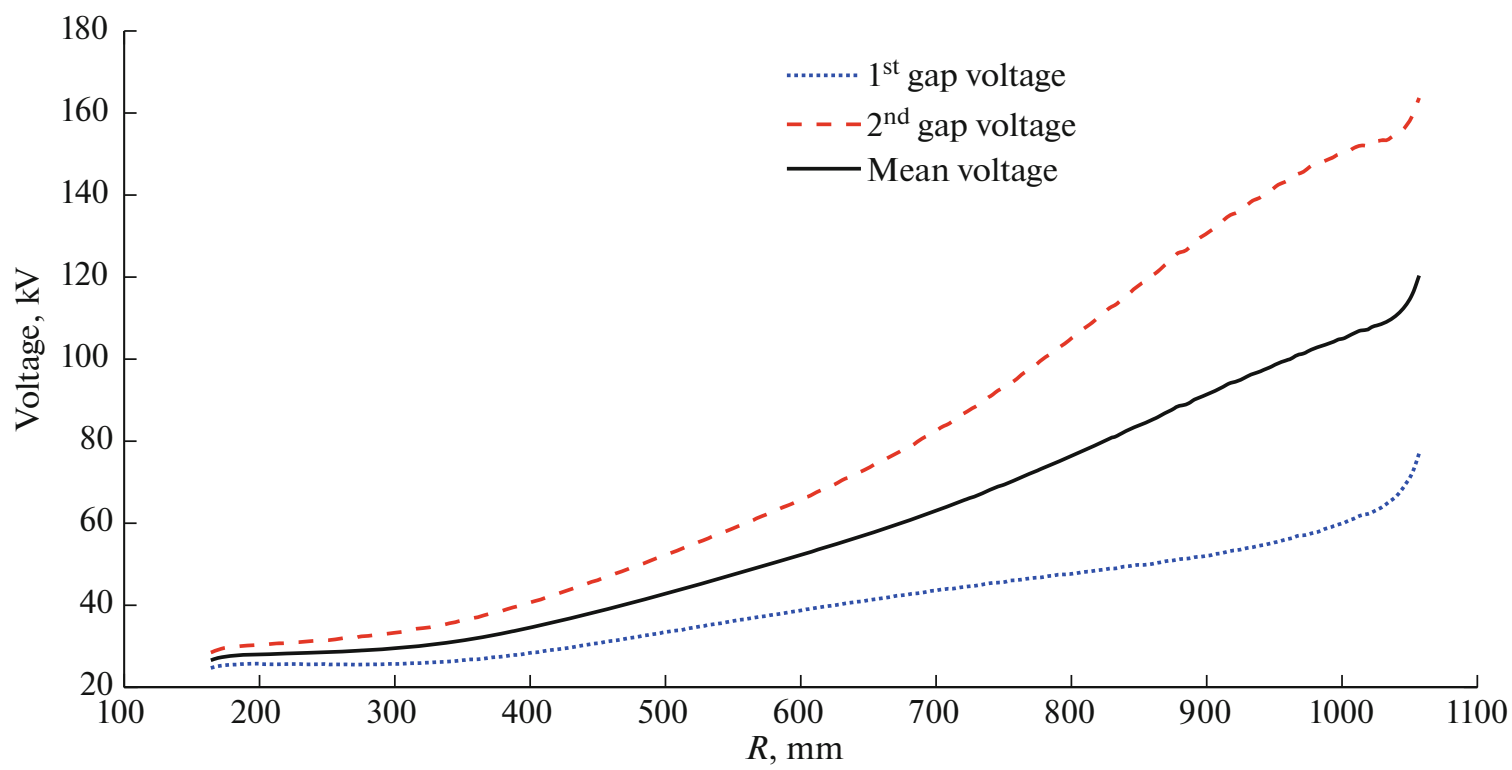

Fig. 9. Changes in the accelerating voltage along the radius.

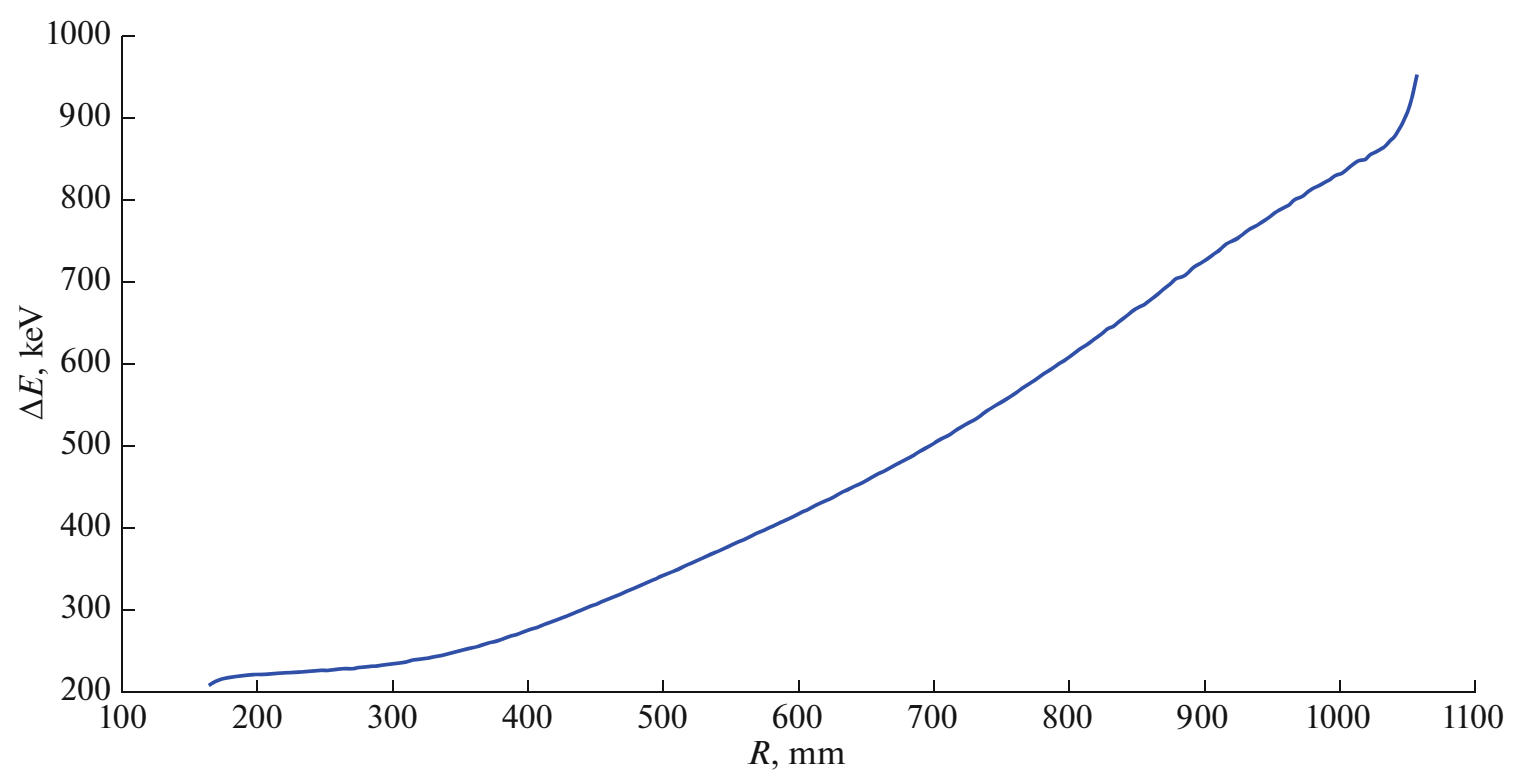

Fig. 10. Dependence of the maximum energy gain per revolution on the radius.

information about the possibility of beam acceleration only based on the analysis of closed equilibrium orbits, without requiring tracing of the beam through the acceleration zone. Green lines in Fig. 11 were obtained from the calculation of the beam acceleration in the cyclotron. Calculation of the beam motion in the acceleration zone is necessary to analyze the effect of resonances; Fig. 13 shows no increase in the vibration amplitudes during acceleration.

Power losses in the accelerating and magnetic systems. The dissipated power in the model was calculated based on the fact that the resonator walls consist of copper with conductivity $\sigma=5.8 \times 10^{7} \mathrm{Ohm}^{-1}$. The quality factor of the HF system was about 13800 , and the power loss in four resonators was $55 \mathrm{~kW}$, while the voltage in the center/extraction zone was $30 / 120 \mathrm{kV}$ (Fig. 9). The calculation showed that the general requirements for the power and cooling of the HF system are less than for C235 (IBA) and significantly less than for the Varian cyclotron.

Beam extraction. The beam will be extracted from this cyclotron using an electrostatic deflector (ESD), two passive magnetic channels, and two compensating channels. The axial gap between the sectors is $50 \mathrm{~mm}$, 


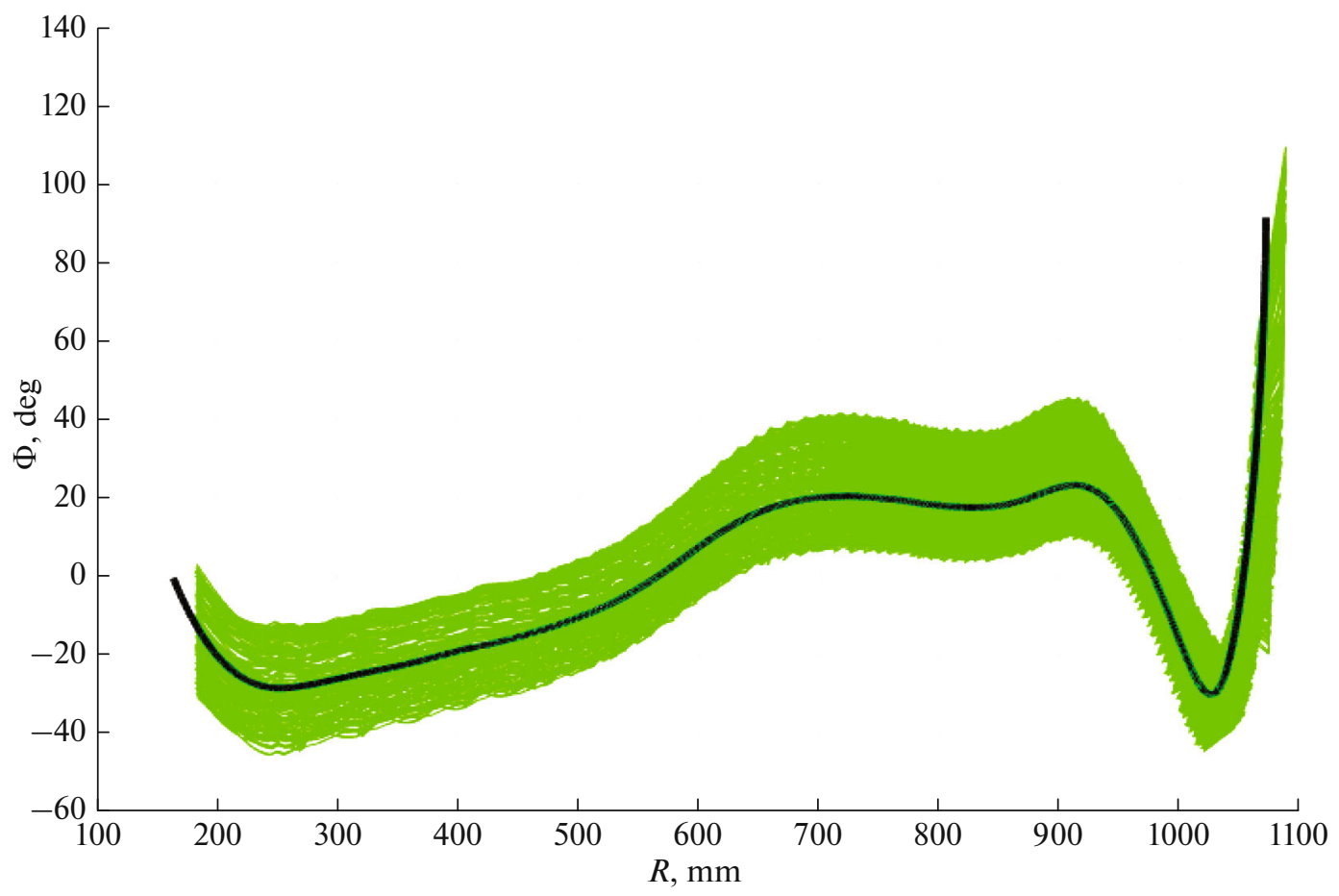

Fig. 11. Integral phase shift depending on the radius.

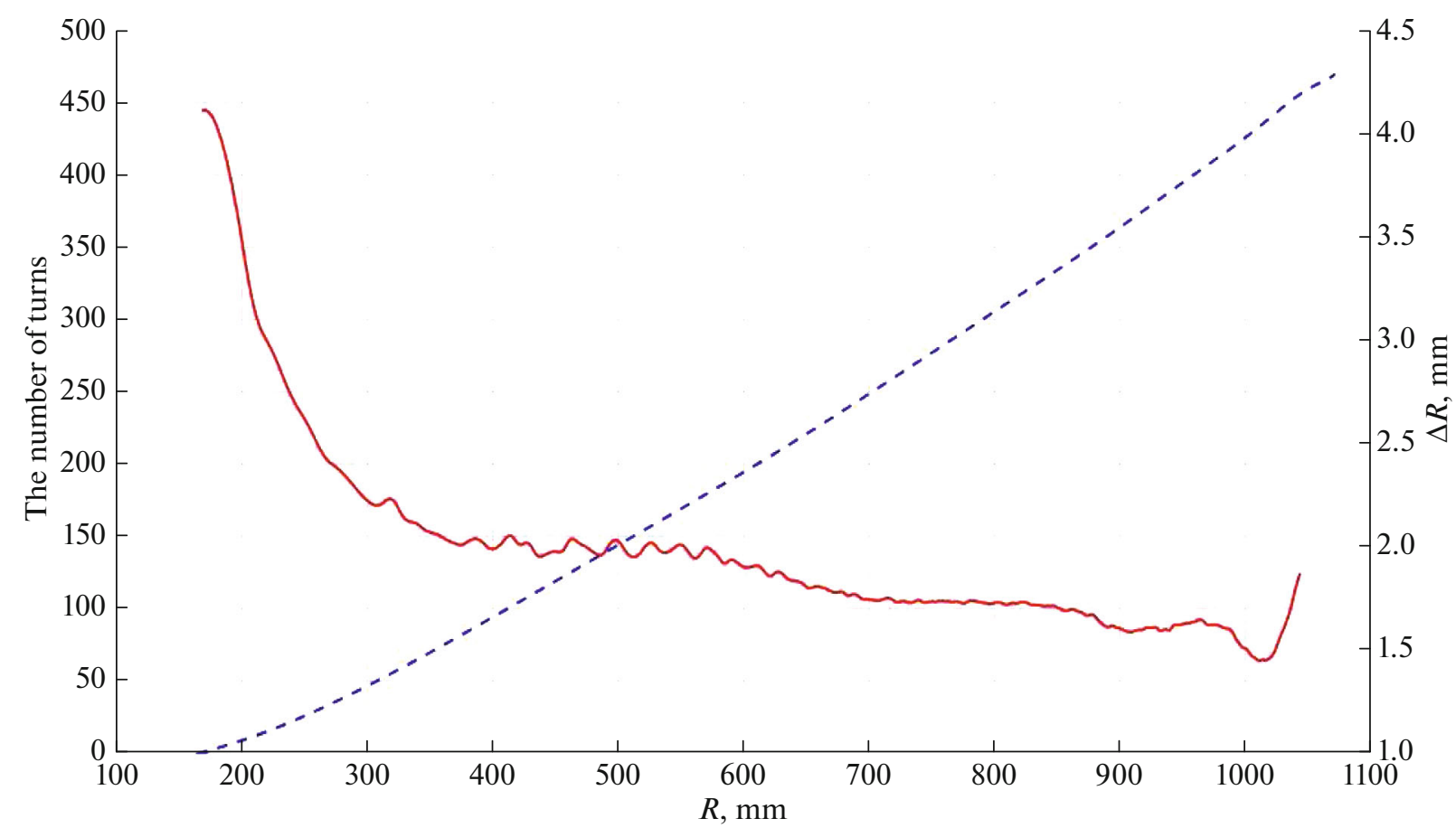

Fig. 12. Changes in the number of revolutions (blue dashed line) and the step of the orbits (red solid line) in the cyclotron along the radius.

which allows accommodating an electrostatic deflector. The voltage across the ESD required for beam extraction is only $90 \mathrm{kV} / \mathrm{cm}$. After going through the deflector, the beam passes through the accelerating cavity and leaves the cyclotron according to the scheme developed previously for extracting the beam 

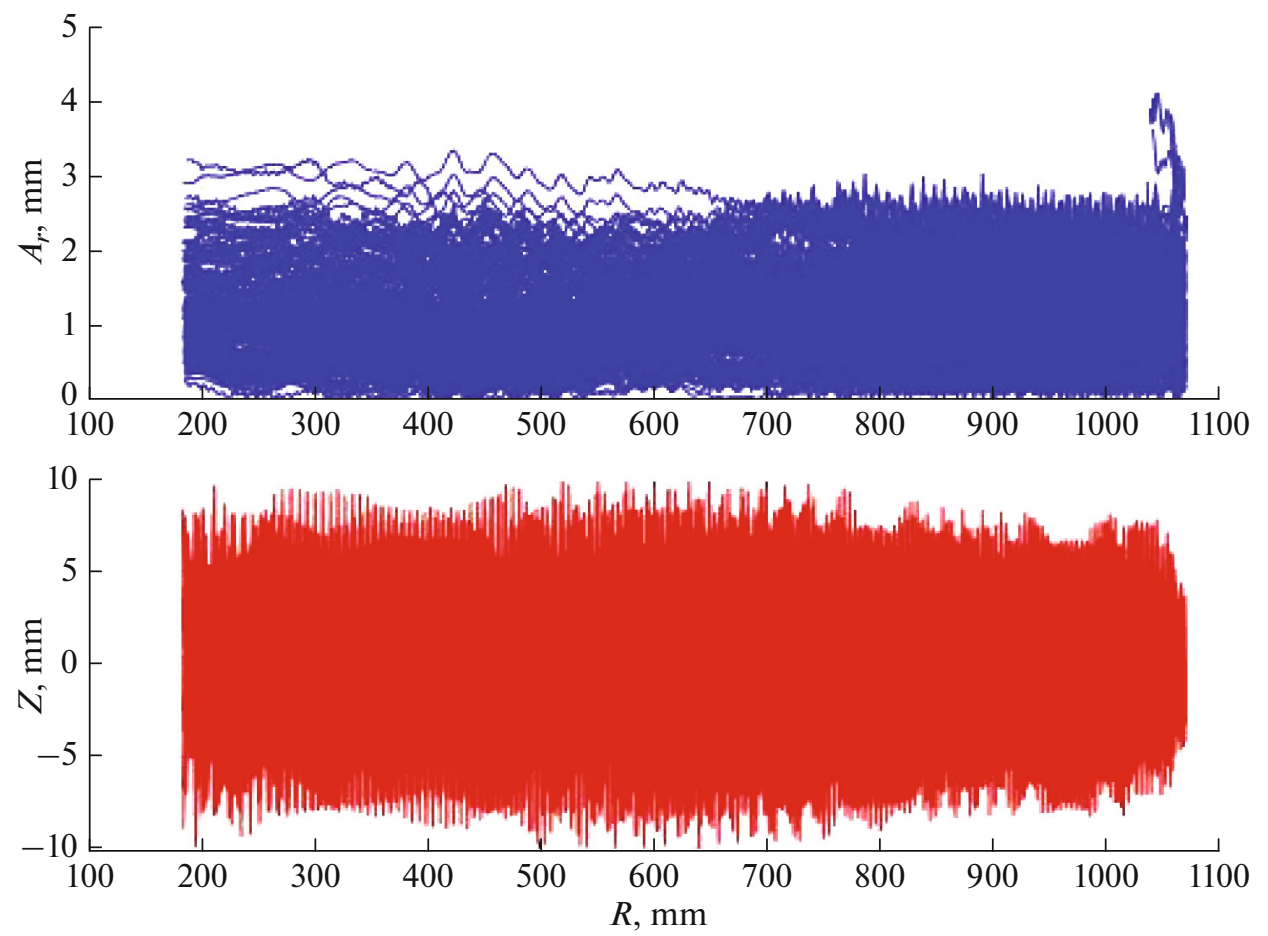

Fig. 13. Amplitude of radial oscillations of the beam (top) and axial motion (bottom), depending on the radius.

from the SC200 cyclotron [19]. Passive magnetic channels are located along the sectors, the first magnetic channel introduces a change in the magnetic field of $600 \mathrm{G}$ and a gradient of $1000 \mathrm{G} / \mathrm{cm}$, and the second magnetic channel introduces a gradient of $1700 \mathrm{G} / \mathrm{cm}$.

\section{CONCLUSIONS}

Selecting a low level of the magnetic field in the cyclotron, we found that the dimensions and weight of the cyclotron can be small if the parameters and geometry of the accelerator are carefully adjusted. An accelerator with such parameters can become the most energy-efficient accelerator for proton therapy due to the low power consumption of both the accelerating system and the superconducting coil.

The SC230 superconducting cyclotron is an excellent candidate for implementing the medical research program at JINR and for use as a prototype in the serial production of medical cyclotrons intended for application in full-scale proton therapy centers. This accelerator is also an interesting candidate as a device whose intense beam can be used in the new FLASHRT method, since the spacious central area allows accommodating two internal sources or organizing the axial external beam injection by means of a spiral electrostatic inflector.

This project incorporates conservative and proven solutions that reduce risks and make engineering sim- pler, as well as innovative solutions, such as a noncircular shape of the coil, which significantly reduces the overall dimensions and weight of the cyclotron.

The low power consumption of the magnet coil makes this project attractive for the use of high-temperature superconductors.

\section{ACKNOWLEDGMENTS}

The study was carried out at the Dzhelepov Laboratory of Nuclear Problems (JINR).

\section{REFERENCES}

1. O. V. Savchenko, " 40 years of proton therapy on synchrocyclotron and phasotron of LNP, JINR," J. Med. Phys., Nos. 3-4 (2007).

2. E. V. Syresin et al., "Development of radiation medicine at DLNP, JINR,” Phys. Part. Nucl. Lett. 8, 635646 (2011).

3. R. V. Galkin, S. V. Gurskii, Y. Jongen, G. A. Karamysheva, M. Yu. Kazarinov, S. A. Korovkin, S. A. Kostromin, J.-M. Calderan, P. Cahay, S. P. Mokrenko, N. A. Morozov, H. Nkongolo, A. G. Ol'shevskii, Y. Paradis, D. S. Petrov, et al., "C235-V3 cyclotron for a proton therapy center to be installed in the hospital complex of radiation medicine (Dimitrovgrad), "Tech. Phys. 59, 917 (2014).

4. Y. Jongen et al., "Compact superconducting cyclotron C400 for hadron therapy," Nucl. Instrum. Methods Phys. Res., Sect. A 624, 47-53 (2010). 
5. A. Patriarca, "FLASH radiation therapy: Accelerator aspects," in Proceedings of the 11th International Particle Accelerator Conference IPAC 2020, Caen, France.

6. S. Jolly et al., "Technical challenges for FLASH proton therapy," in Proceedings of the International Conference on Medical Accelerators and Particle Therapy, Seville, Spain, 2019.

7. G. Karamysheva et al., "Present status of the SC202 superconducting cyclotron project," in Proceedings of the 8th International Particle Accelerator Conference (IPAC 2017), Copenhagen, Denmark, 2017, THPVA120.

8. V. Smirnov et al., "The R\&D of a K230 superconducting cyclotron for proton therapy," in Proceedings of the Conference ECPM 2018, Dubna, 2018.

9. H. Tsutsui et al., "Status of Sumitomo's superconducting isochronous cyclotron development for proton therapy," in Proceedings of the 13th International Topical Meeting on Nuclear Applications of Accelerators AccApp'17, Quebec, Canada, July 31-August 4, 2017, p. 419.

10. D. Vandeplasche et al., "Extracted beams from IBA's C235," in Proceedings of the Particle Accelerator Conference, 1997, Vol. 1.

11. J. Schippers et al., "The SC cyclotron and beam lines of PSI's new proton therapy facility PROSCAN," Nucl. Instrum. Methods Phys. Res., Sect. B 261, 773 (2007).

12. O. Karamyshev et al., "JINR projects of cyclotron for proton therapy," in Proceedings of the 22nd International
Conference on Cyclotrons and Their Applications, Cape Town, abstract TUB02, pp. 141-144.

13. O. Karamyshev et al., "Conceptual design of the SC230 superconducting cyclotron for proton therapy," in Proceedings of the 10th International Particle Accelerator Conference IPAC 2019, abstract TUPTS059.

14. O. Karamyshev et al., "Conceptual design of the SC230 superconducting cyclotron for proton therapy," JINR Preprint No. E9-2019-2 (JINR, Dubna, 2019).

15. L. Calabretta et al., "LNS Catania project for therapy and radioisotope production," in Proceedings of the 18th International Conference on Cyclotrons and Their Applications, Tokyo, Japan, October 2004, pp. 179-191.

16. M. Gordon, "Computation of closed orbits and basic focusing properties for sector-focused cyclotrons and the design of "Cyclops"," Part. Accel. 16, 39-62 (1984).

17. Y.-N. Rao and R. Baartman, in Proceedings of the 2013 International Workshop on FFAG Accelerators FFAG'13, TRIUMF, Vancouver BC, Canada, September 21-24, 2013.

18. C. Baumgarten, Nucl. Instrum. Methods Phys. Res., Sect. A 647, 31-33 (2011).

19. K. S. Bunyatov et al., RF Patent No. 2702140 (2019).

Translated by K. Lazarev 